\title{
Heterogeneous Remote Visualization Framework for Ubiquitous Product Development Activities
}

\author{
Eliab Z. Opiyo and Imre Horváth
}

Faculty of Industrial Design Engineering, Delft University of Technology, Land berg street 15, 2628 CE, Delft, The Netherlands

\begin{abstract}
Design office, as is known today, is a workplace where various experts gather and develop products. Traditionally, the trend in engineering product development has been to use desktop computers, virtual reality systems and other systems and devices placed in specific workplace locations. Clearly, these sorts of stationary support tools are desirable because they matchup the product development methodological frameworks that have prevailed over the past several decades. With the availability of a wide spectrum of improved mobile and wireless communication technologies - coupled with increased network bandwidth and enhanced software for mobile data-communication networks, design researchers now have new opportunity of exploring and coming up with concrete recommendations on how these technologies should be utilized in product development. Therefore, the work reported in this paper aimed at investigating how to take advantage of these technologies and how the product developers can benefit from the capabilities provided by both portable and non-portable information and communication devices. The focus was specifically on the challenge of visualization of product data and models remotely. The main characteristic requirements for a remote product visualization environment have been formulated and used as the basis for creation of a generic framework. It has been shown that the proposed framework meets some of the key requirements.
\end{abstract}

Index Terms - Virtual reality, distributed product development, remote product data visualization, computer-aided design, ubiquitous product development.

\section{INTRODUCTION}

Visualization plays a vital role in product development. Today, stationary visual display units are predominantly used in many organizations involved in product development. These include standard flat screen visual display units such as CRT displays, liquid crystal displays and plasma displays widely used for visualization of product data and models generated by Computer Aided Design (CAD) systems. Three-dimensional (3D) visualization devices such as 3D glasses and Head Mounted Displays (HMDs) are also widely used in Virtual Reality (VR) systems to visualize product models. HMDs are

Manuscript Received on March 1， 2011

E-mail: E.Z.Opiyo@tudelft.nl typically worn on the head or as part of helmet, and have small display optics (i.e., lenses or semi-transparent mirrors) in front of each eye to filter images and create the 3D effect [1,2].

Apart from the above mentioned mainstream visualization technologies, there are several other emerging technologies. These include: auto stereoscopic displays, which enable viewers to experience 3D views without using 3D glasses, HMDs or other stereo view enhancing devices [3,4]; aerial display systems such as Heliodisplay (http://www.io2technology.com) and Fog Screen ${ }^{\mathrm{TM}}$ display (www.FogScreen.com), which generate images on aerial projection screens in mid-air; enclosed volumetric displays, which use techniques such as swept-volume technique (i.e. sweeping two-dimensional (2D) image through spatial volume at high frequency) and static-volume technique (using self-luminous or light reflecting medium to generate 3D image in enclosed volume of space) [5]; and holographic displays, which produce 3D images by reproducing diffraction of light from 3D scenes [6,7]. Refer to [8] for a comprehensive review and analysis of the existing and emerging visualization technologies. All these types of visualization technologies are usually placed and used in fixed office locations. In addition to the displays-based methods of product visualization, engineering technologies such as selective laser sintering (SLS), fused deposition modeling (FDM), stereo lithography (SLA), laminated object manufacturing (LOM), electron beam melting (EBM), die casting and 3D printing (3DP) are also widely used to produce physical models or prototypes. Just like computer-based virtual models, physical models produced by using these engineering technologies also allow the product developers to visualize and evaluate their designs.

With the availability of a wide spectrum of new handheld wireless data communication technologies, a paradigm shift from visualizing and interacting with product data and models entirely within the offices by using non-portable visualization technologies to using combinations of portable and traditional visualization technologies is almost inevitable. Remote product data and models visualization will obviously play important role in this emerging paradigm. The term remote product visualization is used in this paper to refer to the act of accessing and interactively visualizing product data or models remotely. Think of an ideal product development process under the above-described imagined scenario (also depicted in Fig.1). A 


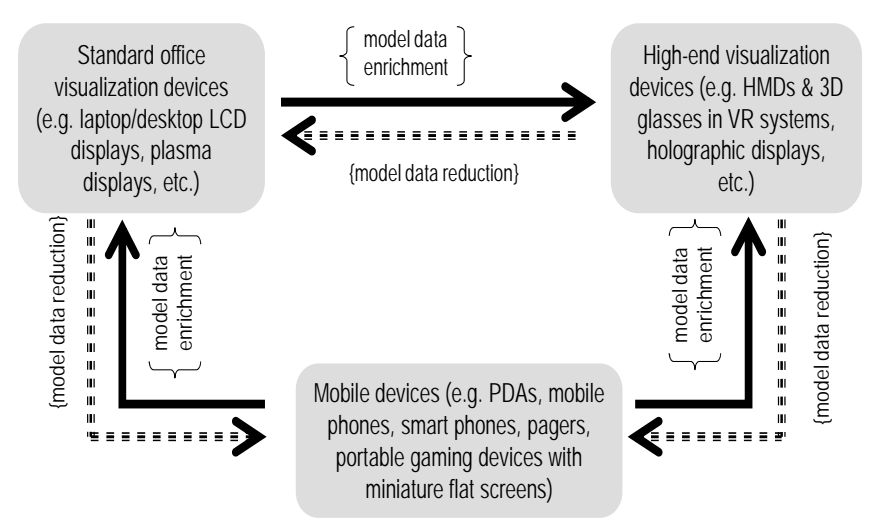

Fig.1. Information loss or enrichment among interconnected heterogeneous visualization devices

designer or an engineer (a viewer) working in an office should be able to design and visualize product data or models by using a high performance computing system located in the office and at the same time communicate and share the product data or models with remotely located team members, who may only be equipped with relatively low performance handheld information and communications devices. There should also be a possibility for remotely located team members equipped with low performance information and communications devices to visualize product data or models as they wish. In general terms, the nature of mobile communication devices such as smart phones and personal digital assistants (PDAs) makes them ideal tools for networking in distributed product development environments, where members of product development teams routinely carry these devices with them and use them for communications. Sophisticated software tools that enable interactions within mobile networks are also nowadays widely available. These software tools not only support text and verbal message exchanges, but are also increasingly developing towards supporting many other sophisticated information exchanges and interactions.

The work presented in this paper is part of a much wider research activity in which the aim is to conceptualize and implement a prototype of the prospective heterogeneous remote product visualization environment. The intention is to come up with an efficient and trouble-free heterogeneous visualization environment that permits two-way communication and delivery of product data and visual information from a non-portable visualization device to a portable handheld visualization device and vice versa. Challenges involved in creating a suitable framework and in realizing a fully operational prototype of the prospective heterogeneous remote visualization environment with the above-described functional characteristic features are enormous. For instance, efforts should be made to ensure that suitable infrastructure (i.e. for networking and visualization) is set up; there must be a comprehensive content management system and a suitable data and information communication and exchange regime, e.g., to ensure that remotely located data can be accessed or shared securely and safely, and without loss of the meaning of data or important product model details; and it is also necessary to ensure efficient communication and interaction with remotely located visualization systems. All in all, what is needed is an environment that provides users access to remotely located product data. Therefore, attempts should be made to answer research questions such as: how visualization during product development can be supported? What kinds of available technologies are best suited for the imagined paradigm? (i.e., what combinations of technologies can best provide the intended support?) Which existing utilities in mobile handheld devices can be adopted and applied? How can the foreseeable challenges such as handling of large data and computational resource limitations be dealt with? How can the diversity of product data, data models and data formats be dealt with? What features should the envisioned environment have? How can seamless integration of underlying devices and technologies be achieved? What networking methods would be suitable? What difficulties the users might possibly face in using these technologies? How will the envisioned remote visualization environment work? The focus of this part of the research was specifically on exploring the state of the art and developing a suitable framework for heterogeneous remote product visualization.

The paper is structured as follows. It first analyses the prevailing remote visualization methods and technologies. It then explains the notion of remote product data and models visualization and presents the key characteristic requirements and features of the envisioned visualization environment. Afterward, it introduces the framework for heterogeneous remote product data and models visualization. The results of the preliminary evaluation carried out to determine the validity of the requirements and the completeness of the proposed framework are also presented and discussed.

\section{ANALYSIS OF THE EXISTING REMOTE VISUALIZATION METHODS AND TECHNOLOGIES}

Several remote visualization enabling methods have been proposed in the past few decades and some of them have already been implemented in commercial systems. The following subsections review and analyze the methods and technologies presently used for data and information sharing, for connecting visualization devices, and for networking.

\subsection{Methods and Technologies for Data and Information Sharing}

The common approach for data and information sharing has been to use either: (i) the existing Web/Internet - based tools, or (ii) customized software applications to log onto remote visualization engines via Web browsers. In most modern practical applications, interconnected networks use the Internet Protocol (IP) and IP based tools such as web browsers and file transfer applications. Apart from the Internet (which consists of worldwide interconnections of public and private networks): (a) networks that are under the control of a single administrative 
entity (called Intranet), and (b) those that are limited in scope to a single organization or entity but which also have limited connections to the networks of one or more other trusted organizations who participates in them (widely known as Extranet) are also common.

Currently, web-based utilities have widely been adopted as collaboration support tools in product development. They provide the product developers with means to combine multimedia information and to publish relevant information in various stages of the product development process [9]. With the Web, collaborating team members can share product data, information and knowledge remotely [10,11]. Several different Web-based collaborative frameworks have been proposed - see, e.g., $[12,13,14,15,16,17,18]$. Most of them have been implemented using Java, while Hyper Text Markup Language (HTML), Java Applets, ActiveX and Virtual Reality Modeling Language (VRML) have predominantly been used for implementing user interfaces - see e.g. [9,19]. Others have been implemented by using programming environments such as Prolog - see e.g. [20] and Common Lisp -see e.g. [21]. In Web-based systems, the data exchange problem due to complexity of CAD systems, varying requirements of organizations using them, restrictions on access to proprietary database information, and the problem of incompatibility of hardware platforms is eased by using neutral representations such as VRML [12,22,23]. Furthermore, these systems usually use three-tier architecture, often with techniques such as JDBC, SQLJ and ODBC used for sharing information over the web and for integrating databases [9,17]. Apart from using the existing IP-based tools for accessing product data remotely, a different way of accessing product data remotely, which involves using customized software to log onto a remote visualization engine via a Web browser, is also common. One example is Sun(TM) visualization system (http://www.sun.com/), which integrates workstations, servers, networks, graphics, and software applications to deliver 3D visualization on a variety of devices.

\subsection{Techniques for Connecting Visualization Devices}

Visualization devices can be connected by wiring or without wiring. Wired networks consist of devices interconnected by using different kinds of media such as coaxial cables, fiber optic cables, and twisted-pair cables (e.g. in phone lines and power lines). Wired networks characteristically have higher speeds. By contrast, wireless networks use wireless transmission medium. Examples of the existing wireless networking media and protocols include: terrestrial microwave; communications satellites (which use microwave radio as their telecommunications medium); cellular and Personal Communication Service (PCS) systems (which use radio waves and other communications technologies); and wireless dedicated local area networks (which use high-frequency radio technologies). Others are Bluetooth (an open wireless protocol for data exchange over short distances of $10-100$ meters); infrared signals, and wireless web (i.e. use of the World Wide Web through equipment like cellular phones, PDAs, and other portable communications devices to provide anytime/anywhere connections). The devices themselves can be connected logically and/or physically in different layout configurations. For instance,wired devices can be connected in point-to-point, bus, star, tree (or hierarchical), ring, star-bus, or mesh layout, depending on the specification requirements on ground. The interconnections can be implemented in different scales, e.g. as Local Area Network (LAN), Wide Area Network (WAN), Metropolitan Area Network (MAN), Personal Area Network (PAN), Virtual Private Network (VPN), or Campus Area Network (CAN). Ad hoc networking and sensor network architectures are also increasingly used to enable devices to communicate directly [24].

\subsection{Networks and Networking Methods}

There are many ways by which multidisciplinary teams in various geographic locations can communicate, interact and share information. Communications can be made through a computer network, Internet network, or through a telephone network. In terms of functional relationships that exist among the elements of the network and applications, networks and networking methods can be categorized as P2P and mobile networking (Fig. 2) - see also e.g. [25]. P2P or peer-to-peer network architectures typically have nodes that make portion of resources such as processing power, storage space, and network bandwidth available to peers without intermediary network hosts or servers. Each node in the network can function simultaneously as a client and as a server [26]. P2P networks architectures can further be classifies as: (a) client/server architecture; (b) centralized P2P network architecture; (c) decentralized P2P network architecture; and (d) hybrid P2P network architecture - refer to [25, 26, 27] for further details.

By contrast, mobile networks use several different techniques for enabling communication. For instance, some existing mobile networking applications use Wi-Fi enabled laptops, cell phones or PDAs to link up to other devices within walking distances [28]. Location-aware interaction systems also enable handheld device users to hook up with each other [29]. There are also mobile P2P networking and file-sharing systems are basically P2P systems created specifically for wireless mobile networks. Refer, e.g., to [30] for further details of mobile peer-to-peer. Overall, mobile social networking is

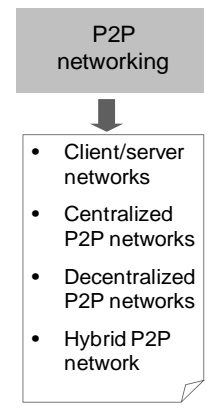

(a) Types of P2P networks

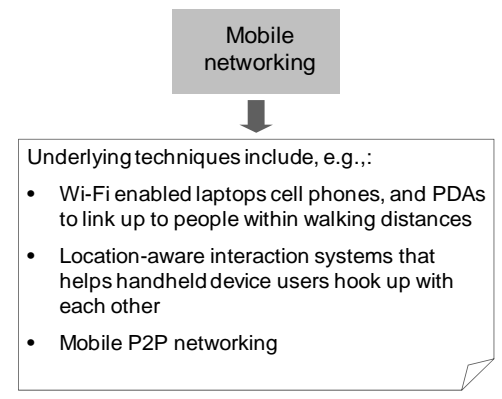

(b) Mobile networking techniques
Fig. 2. Networks and networking methods. 
nowadays a popular way of social networking where individuals with similar interests connect with one another using mobile phones in virtual communities [25].

\subsection{Summary}

In the light of the above review and analysis, it can be said that IP-based tools and customized software applications offer two different possibilities for users to linkup to different devices and applications remotely. In both approaches, software allows a viewer to log onto a remote visualization engine via wireless network or wired network (e.g. Ethernet cable network, Power line Digital Subscriber Line - PDSL, optical fiber cable networks, etc.), and use its resources, including graphical resources. Wireless networks are known to have some limitations that may affect their robustness in supporting remote product visualization. For instance, they are typically slower than wired networks enabled, e.g., through Ethernet cables. Also, wireless networks are generally more vulnerable, because anyone can try to break into a network broadcasting a signal. Although networks often offer security systems which block some intruders, wireless networks have still been vulnerable to intrusion. To achieve a reliable and a robustly performing remote product visualization environment, apart from network speed and security concerns, attempts should also be made to ensure that the envisioned product visualization environment meets some specific functional demands. The following Section describes the desirable features and functional characteristics of the envisioned environment, and introduces the solution concept.

\section{DESIRABLE FEATURES AND SOLUTION CONCEPT}

A remote product data visualization environment is regarded in this work as a system of interconnected dissimilar display devices used to visualize product data and models (see Fig. 1). Some of these dissimilar displays may be at different geographical locations. Fig. 3 shows examples of product data and visual information that would be handled by the envisioned product visualization environment. The idea is to support remote participation in product development processes by integrating workstations, servers, and wireless handheld devices to enable both 2D and 3D visualization on a variety of displays embedded or connected to these devices. The intention is to embrace the application of handheld portable devices in product development processes and in this way improve flexibility in the execution of processes, improve analytical and innovation ability of product developers, to heighten productivity, and to speed up time needed to finalize design and other product development activities. Such an environment should first and foremost fulfill the need for supporting modern-day collaboration of individuals with similar interest and equipped with different types of portable computing and communication devices or non-portable visualization platforms, who may as well be at different geographical locations.

Through the analysis of the existing collaboration and data exchange techniques, analysis of product data and visual information used in product development, literature studies, review of marketing documents of various visualization technologies; and through imagination and reasoning, the following requirements, which describe the characteristic features of the envisioned remote product data and models visualization environment were formulated:

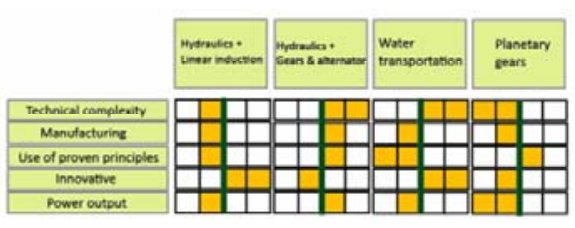

(a) Concepts comparison and selection

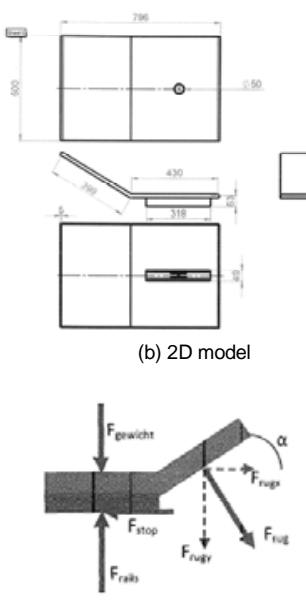

(c) Free body diagram

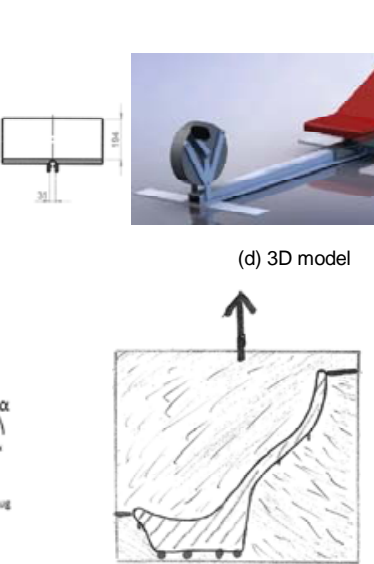

(e) Hand sketch

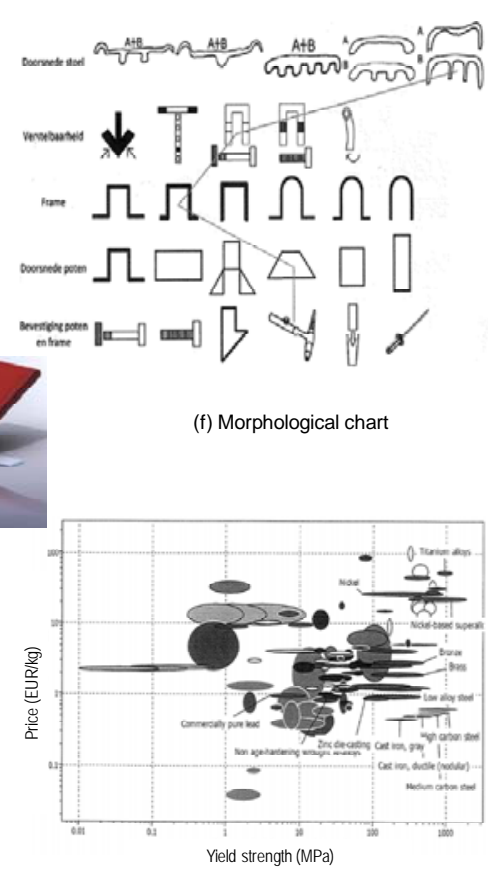

(g) Materials properties and price information

Fig. 3. Examples of visual representations that could be viewed remotely. 
- $\quad$ R1: The visualization systems must be able to remotely connect, adapt, and access product data; and the environment should be built based on the existing networking standards and protocols. The prospective environment should be well suited to product visualization in ubiquitous context.

- $\quad$ R2: If a user equipped with a handheld portable device wants to perform a computationally intensive task (e.g., long and complex computations that would expend precious limited computing resources and battery power), that task should be performed on a remote high performance non-portable device instead of a portable device. The framework and infrastructure of the envisioned environment should allow this.

- R3: Graphics rendering and visualization should not be problematic, namely, local rendering systems should be equipped with suitable interfaces and be capable of displaying 2D, 2.5D or 3D product models.

- R4:A user (a viewer) should not be constrained by the capabilities of locally available computing and graphics resources. Mechanism for enabling users to access and apply tools and resources available elsewhere must be incorporated. Applications must be both sharable and efficient.

- $\quad$ R5: The environment should facilitate interactive remote visualization. A user, who may be at a remote location, should be able, e.g., to interactively view geometric representations of products, design history and so forth.

- $\quad$ R6: During team discussions, each participant must always be able to view shared data or product models. For instance, features of the product model used in a discussion session must be visible to each participant. And the dynamic effects of modifications made by one participant must be visible to all other participants as well.

- R7: Viewers should be able to see both the model (separately) and the scene (model \& people around it including their actions and gestures), and also to listen to verbal communications.

- $\quad$ R8: The user must be able to archive product data after modifying a product model or a design.

- $\quad$ R9: The envisioned environment must be equipped with algorithms for processing, modeling and converting product data into the representations that can be handled by the interconnected visualization devices. These algorithms must be operable by using any device connected to the network. It should be possible, e.g., to create 2D images or $3 \mathrm{D}$ scenes and vice versa in real-time on a handheld device, and so forth.

- R10: Mechanisms for handling large data and for data size tailoring must be incorporated into the envisioned environment.

- $\quad$ R11: Security is a major concern for product data that will be transferred between handheld portable devices and base servers like desktop or laptop PCs. There should not be any security concerns.

- $\quad$ R12: The envisioned environment must be equipped with suitable mechanisms for managing and ensuring efficient usage of limited resources such as storage space and CPU/GPU in handheld devices.
- R13: Exchange of visual information (images) should not lead to any miscommunication. Information context must remain intact. It should be possible to carry over display contents from one display device to another, but the meaning of data should not change.

- R14: Users must be able to transform product models. It should be possible to change models to any form or format suitable forever y visualization device in the network.

- R15: The applications server of the environment must provide any desired content or access to applications to any visualization system requesting a service.

Before the visualization environment is fully designed, more specific and elaborate requirements that describe these general statements in more concrete terms must be formulated. Fig. 4 shows the solution concept proposed for realization of the envisioned heterogeneous remote product visualization environment. The two main categories of software resource needed for the heterogeneous remote visualization environment are: (i) system resources, and (ii) problem solving resources. System resources are utilities within the visualization environment that support operations such as task management, information storage, and information retrieval. By contrast, problem solving resources are device level utilities that enable the product developer to use visualization resources of any device in the network to interactively visualize product data or models. The following section describes the main infrastructural elements of the envisioned environment.

\section{COMPONENTS OF THEHETEROGENEOUS REMOTE PRODUCT MODEL VISUALIZATION ENVIRONMENT}

The two core infrastructural elements of the proposed heterogeneous remote product data visualization environment are: (1) visualization platforms, and (2) communication networks. Fig. 5 shows the layout of the main functional devices.

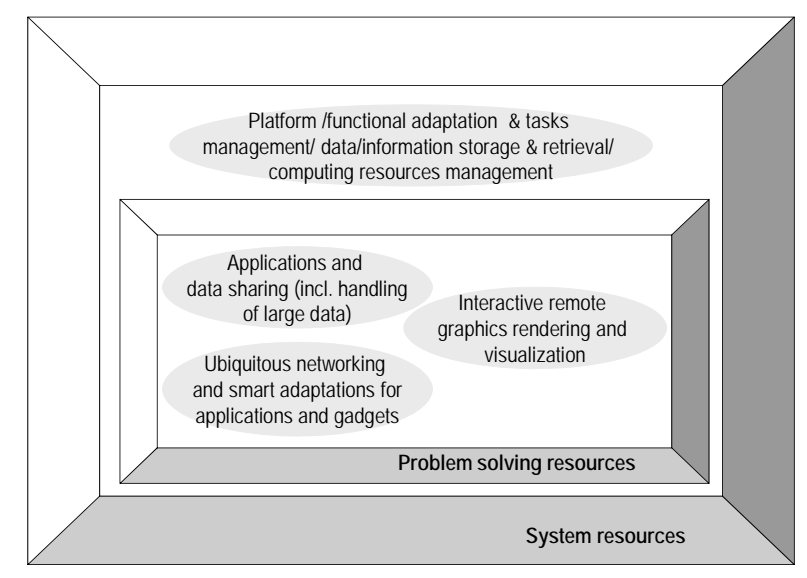

Fig. 4. Solution concept for realization of heterogeneous remote product visualization environment. 


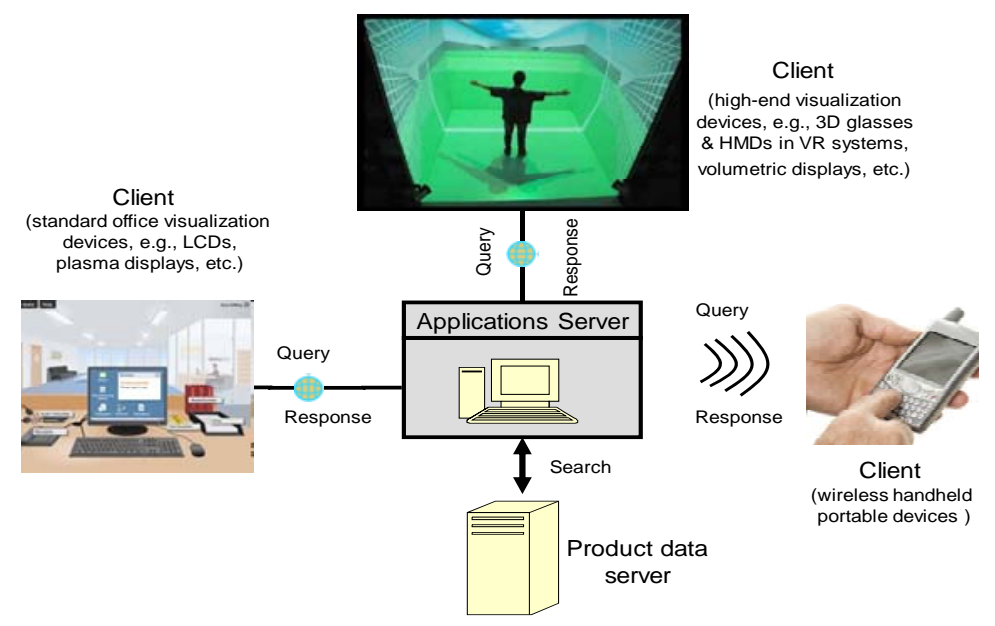

(a) Layout of functional devices

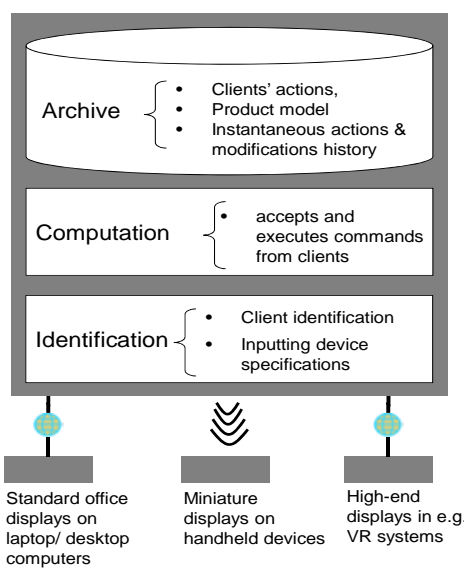

(b) A conceptual structure of the applications server

Fig.5.Elements of the remote product data visualization environment.

\subsection{Visualization Platforms}

Desktop and laptop computers, wireless handheld portable devices and high-end visualization systems provide the graphics rendering and visualization infrastructure for the proposed heterogeneous remote product data visualization environment.

\section{1) Standard Office Based Visualization Devices}

Desktop and laptop computers are traditionally equipped with flat screen displays - in most cases liquid crystal displays, plasma displays, or CRT displays. Flat screens have long been the familiar visual display units for CAD systems and are among the visualization platforms of the envisioned environment. Standard CAD systems still rely heavily on these 2D display technologies for displaying both 2D and 3D images. These displays serve primarily as passive visual information output devices. Interaction with the displayed virtual models is mainly possible by using the traditional input devices such as keyboard and mouse; via the traditional Windows, Icons, Menus and Pointing device (WIMP) style graphical user interfaces. Apart from being equipped with flat screen displays, desktop and laptop computers typically have powerful CPUs and GPUs that carry out instructions and process information, and also manipulate graphics. Utilities such as microphones and speakers are integral parts of standard office-based computing and communications systems. These utilities would play important roles in remote product visualization.

\section{2) Miniature Displays on Wireless Handheld Devices}

Wireless handheld devices such as smart phones and PDAs are also the key infrastructural devices of the envisioned heterogeneous remote product data visualization environment. Table 1 shows some utilities embedded in modern wireless handheld devices and their potential applications. It can be seen that most of the utilities available on desktop and laptop computers such as flat screen displays, memory and CPU/GPU are also available on mobile wireless handheld devices. The only significant distinction is that in most instances handheld portable devices have scaled-down capabilities and/or sizes. For instance, these devices have comparatively limited heap space and storage space, and some of them do not have disk drives or PCMCIA card support. Removable memory is, however, provided in some devices to expand internal memory so as to allow users to store large data files such as audio files, photos and video files separately from other data. Memory cards are available in various sizes, with adapters for laptops, digital cameras and other digital devices.

In general terms, wireless handheld portable devices require lean operating systems and applications. Application codes must be organized for efficiency within a small address space. Also, most modern wireless handheld devices are equipped with extra utilities such as cameras (i.e. for taking still pictures and for recording or playing video), which are not commonly available on standard office based computing equipment. These sorts of utilities would be useful to the envisioned remote product data visualization environment. All in all, there is an increasing popularity and growing application of mobile computing in day-to-day activities, and the widespread demand for multifunctional mobile devices, from mobile and smart phones to portable gaming systems and PDAs is driving amazing mobile devices hardware and software applications developments. These advances would obviously be beneficial in the realization of prototype of the prospective remote product visualization environment.

\section{3) High-end Visualization Systems}

$3 \mathrm{D}$ visualization technologies such as HMDs and 3D glasses used in VR systems or even some emerging visualization systems such as holographic displays placed in specific office locations will provide high-end visualization functionality for the heterogeneous remote product model visualization environment. High-end visualization in this case involves using devices and systems capable of generating images with depth 
TABLE 1. IMPORTANT UTILITIES IN MODERN WIRELESS HANDHELD DEVICES AND HOW THEY COULD POSSIBLY BE APPLIED.

\begin{tabular}{|c|c|c|}
\hline \multicolumn{3}{|c|}{$\begin{array}{c}\text { Equipment: Mobile phones/ smart phones, (i.e. high-end mobile phones) personal digital assistants/portable gaming devices, } \\
\text { pagers, etc. }\end{array}$} \\
\hline Function & Description & Potential application \\
\hline \multirow[t]{2}{*}{ Input } & $\begin{array}{l}\text { Miniature QWERTY type keyboard (physical or } \\
\text { virtual keyboard) }\end{array}$ & \multirow{2}{*}{$\begin{array}{l}\text { Text input \& menu selection. Smaller mobile } \\
\text { devices such as PDAs, mobile phones, pagers and } \\
\text { smart phones typically use the WIMP elements } \\
\text { with different unifying metaphors, due to } \\
\text { constraints in space and available input devices }\end{array}$} \\
\hline & Stylus \& touch screen/ joystick/thumbwheel & \\
\hline \multirow[t]{2}{*}{ Output } & $\begin{array}{l}\text { Liquid crystal/plasma display (some are } \\
\text { touch-sensitive) }\end{array}$ & Displaying visual information \\
\hline & $\begin{array}{l}\text { Audio utilities (speakers, microphones, MP3 } \\
\text { players, voice recorders) }\end{array}$ & Voice or speech input and output \\
\hline \multirow[t]{2}{*}{ Processors/memory } & CPU/GPU & Data and visual information processing. \\
\hline & Memory & Data storage \\
\hline $\begin{array}{l}\text { Software/ } \\
\text { functionality } \\
\text { envelope }\end{array}$ & $\begin{array}{l}\text { Functionality envelope include personal organizers, } \\
\text { calculators, notepad, word processing, spell } \\
\text { checking, dictionary, spreadsheet }\end{array}$ & Text editing, design related computations \\
\hline \multirow[t]{5}{*}{$\begin{array}{l}\text { Communication/Con } \\
\text { nectivity }\end{array}$} & $\begin{array}{l}\text { Wireless capabilities (mainly Bluetooth\& Wi-Fi } \\
\text { connectivity) }\end{array}$ & Support communication/connectivity \\
\hline & USB2/Blue tooth & Data exchange over short distances \\
\hline & $\begin{array}{l}\text { Internet client (for web browsing, faxing and e-mail } \\
\text { support with POP3/IMAP4/SMTP) }\end{array}$ & Information/data exchange \\
\hline & $\begin{array}{l}\text { Integrated data capture devices like Bar Code, } \\
\text { Radio-frequency identification (RFID) and Smart } \\
\text { Card readers }\end{array}$ & Data capture \\
\hline & Global Positioning System (GPS) & Determining location and orientation \\
\hline \multirow[t]{2}{*}{ Imaging } & Camera & Recording still images \\
\hline & Video & Recording video images \\
\hline
\end{tabular}

information (i.e. 3D images), which allow product developers to explore 3D models, for instance, by looking around the displayed images. In the envisioned environment, these sorts of visualization devices and systems would also be important because they add extra width to the visualization process. They will be used, for instance, in engineering visualization activities that require wide field of view such as visibility and aesthetics studies on, e.g., exteriors or interiors of the product models, reach investigations, review of assembly variations, and exterior or interior ergonomics assessments.

\subsection{Communications Network for Heterogeneous Remote Product Model Visualization}

The communications network for the envisioned heterogeneous remote product visualization environment would be based on the existing networking and communications infrastructure. This is necessitated by the fact that readily available technologies on the market such as portable handheld devices and the existing networking technologies and networks will be adopted and used as building blocks of the environment. Naturally, the associated underlying methods and techniques presently used to link the available devices should also be adopted and used to support heterogeneous remote product visualization. The communications between visualization devices or systems would be made through computer networks, internet network, or through telephone networks. And the technologies used to enable laptops, cell phones, or PDAs to link up such as Wi-Fi and Bluetooth, as well as location-aware interaction systems (which help handheld device users hook up with each other)would play important roles in supporting heterogeneous remote product visualization.

A conceptual structure of the heterogeneous remote product data visualization environment is presented in the subsequent Section. It is important to mention that the final architecture and design of the visualization environment will obviously depend on the devices that make up the environment and on various specific factors. Factors such as performance, existing and future application possibilities, speed, reliability, security and cost should be taken into consideration. The design must also reflect other specific technical considerations such as the band or bands in which the network operator is transmitting - which limit the choice of network architecture.

\section{FRAMEWORK FOR REMOTEVISUALIZATION}

A framework for the envisioned heterogeneous remote product data visualization environment is introduced and discussed in this Section. The proposed framework is based on the general three - tier client-server architecture shown in Fig. 5 (a) and elaborated in Fig. 6. It provides a basis for building an integrated visualization platform that enables collaboration and access to remote visual information and applications across remote data stores and computing devices.

In principle, this framework can be implemented as a 


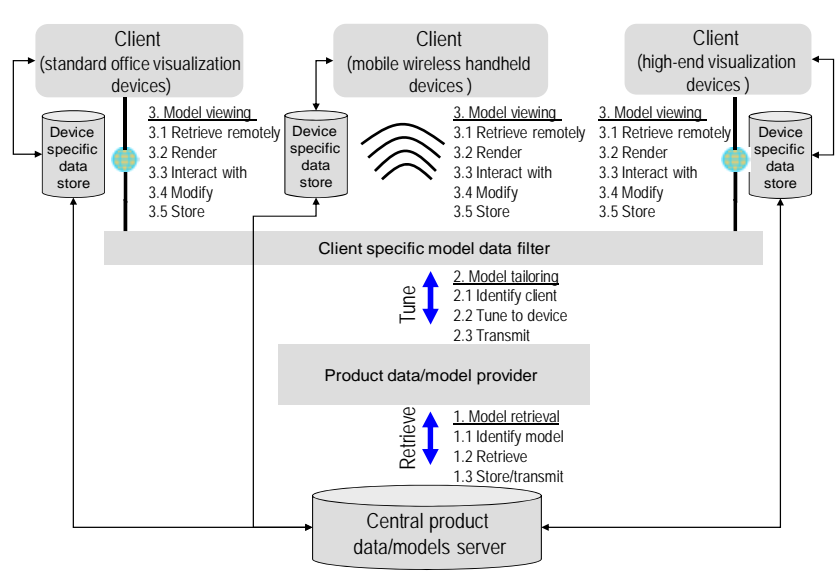

Fig.6. Heterogeneous remote product model visualization framework.

distributed computing and networking environment that partitions tasks between the service providing device (which in this case is a central applications server) and service requesting devices (which in this case are various visualization platforms). The service requesting heterogeneous visualization devices and the applications server operate over a computer network or the Internet network. Under this framework, there is an intermediary service level, meaning that the framework is split up between: (a) a service requesting device (i.e., the computer or wireless handheld device, which requests data or resources), which would typically be equipped with a user interface that could be, e.g., a web browser - for presentation and interaction purposes; (b) the applications server - (which is a central powerful computer whose task it is to provide the requested resources or product data -from the product data server); and (c) the product data server (which provides the applications server with the data it requires, which it ultimately turns, e.g., into images). The applications server is essentially a high-performance computer that hosts and runs applications, and also shares its resources with service requesting devices. Under the proposed framework, a service requesting device does not share any of its resources, but requests the applications server's content or service. Service requesting visualization devices therefore initiate communication sessions with the applications server which in turn listens and handles various incoming requests.

The product data server is intended primarily for storage of product models and other types of product data whilst the applications server is intended for execution of resource-intensive operations (such as modeling and data conversion). In the proposed conceptual framework, the applications server consists of utilities for: client identification, computation, and archiving - see Fig. 5(b). The identification utility allows various users equipped with different types of visualization devices to identify themselves and to log onto the visualization environment. The computation utilities accept and execute queries from service requesting devices while the archive stores product data and models (in suitable file formats), instantaneous actions and modifications history (e.g., in the form of recorded video footages) and other information (such as annotations made the models by participants, gestures used, verbal communications, and so forth).

The proposed framework allows for product data or models heterogeneity in that product data or models can be transformed into any form required by any visualization device or system in the network. Each viewer would be able to visualize information by using a chosen device, interact with product models, and at the same time know what is happening around (e.g., see gestures and listen to verbal conversations). Rendering will obviously depends on the computational resource requirements for the task at hand, namely: (a) images will be generated remotely on a high-performance computing device before being transferred to a low-performance wireless mobile handheld device or, (b) images will first be generated on a low-performance a wireless mobile handheld device before being transferred to a high-performance computing device. Either way, only the resulting images would be transferred.

\section{IMPLEMENTATION AND FUTURE WORK}

Advances in computing and information technology areas have led to development of numerous communications and networking technology solutions. Some of these solutions can be adopted and used in the implementation of a prototype of the proposed framework for heterogeneous remote product data visualization. The use of the existing solutions would obviously allow us to spend less time, e.g., in writing plumbing code during the implementation of the prototype of the prospective environment; and more time in refining the envisioned application logic.

Examples of the existing solutions that could possibly be adopted and applied in building the prototype of the prospective heterogeneous remote product data visualization environment include: .NET Framework, Windows Sensor and Location Platform, OpenGL ES and M3G Standards, and mobile processors. .NET framework offers a model-based paradigm and a rich, standards-based architecture for creating data-oriented applications shared across multiple platforms whilst Windows Sensor and Location platform [31] enables computers and applications to adapt to environments. Applications and gadgets can know exactly where they are by using location sensors such as GPS devices and Wireless Wide Area Network (WWAN) radios. This enables them, e.g., to provide more locally relevant content and functionality. OpenGL ES and M3G are standards for cross-platform mobile and real-time 3D graphics for mobile ICT devices. Adaptation of OpenGL for devices like mobile phones, smart phones, gaming devices and PDAs is obviously also beneficial. Mobile processors (e.g., chips such as nVidia mobile processors) and 3D wireless media processors designed specifically to support 3D graphics in mobile phones and other handheld devices and specially designed for low-power operation to conserve battery life can also be useful building technologies for the prototype of the prospective heterogeneous remote product visualization environment.

Handling of large data, limited storage space and device 
synchronization are the main challenges that must be dealt with if satisfactory performance is to be achieved. A possible solution to the problem of handling of large applications and data files could be through using techniques such as trans coding [32,33], which is one of the techniques by which applications and files can be scaled to mobile phones or PDAs applications to allow, e.g., a data object in one representation to be converted into another representation. One way of tackling the problems of limited storage space and synchronization could be to avoid creating files in the traditional ways, and instead breaking data into multiple records (i.e., instead of data being stored in one contiguous chunk). Data can then be stored in memory chunks and grouped into a database. This method is already applied in some PDA systems, e.g., in Palm OS platform - see e.g. [34].

As mentioned earlier, the focus of the work reported in this paper was on exploration of possible solutions and development of a suitable framework. Low level implementation details will obviously depend on the devices selected and used in building the prototype of the prospective environment.

\section{VALIDATION}

The heterogeneous remote product data visualization framework presented and discussed in Sections V and VI and depicted in Figures 5 and 6 represents an initial high-level implementation of the envisioned environment. It has been evaluated and crosschecked to determine whether or not it lives-up to users' expectations. Evaluation must go on continuously, and needs to be carried out not only after realization of the visualization environment fully, but also early on to ensure that the in-process deliverables meet demands and to determine if requirement specifications were correctly spelt out. Evaluation was therefore carried out to determine the validity of the requirements and to assess the completeness of the proposed framework. Methods such as abstract prototyping [35, 36, 37], focus group research [38], expert evaluation [39], and many others can be used to evaluate systems early on and to determine if specifications were correctly determined. Two different approaches, namely: (1) reviewing the framework - to inspect the extent of fulfillment of requirements, and (2) focused group research - to establish users' attitudes towards the proposed heterogeneous remote product visualization concept, were adopted and used in this work. The following sub sections describe the evaluation processes and present the findings of the evaluations.

\subsection{Review of the Proposed Framework}

The characteristic requirements presented and discussed in Section III have been used as benchmarks for inspecting the proposed framework for any violations. Table 2 shows the inspection results. It can be said, however, that at this stage, it was only possible to hypothesize and to speculate that the
TABLE 2. SUMMARY OF THE RESULTS OF THE ASSESSMENT OF FULFILLMENTS OF REQUIREMENTS - KEY: $\bigotimes=$ IMPLIED TO HAVE BEEN COVERED/WOULD BE FULFILLED (GIVEN THE PROPOSED FRAMEWORK IS IMPLEMENTED); $\boldsymbol{\chi}=$ CANNOT BE ESTABLISHED.

\begin{tabular}{|c|c|c|c|}
\hline $\begin{array}{c}\text { ID (See } \\
\text { Section III) }\end{array}$ & $\begin{array}{l}\text { Has been } \\
\text { fulfilled? }\end{array}$ & $\begin{array}{l}\text { Would be } \\
\text { fulfilled? }\end{array}$ & $\begin{array}{l}\text { Existing examples of possible } \\
\text { enabling method/technology }\end{array}$ \\
\hline R1 & $\otimes$ & $\otimes$ & $\begin{array}{l}\text { Networking technologies of the IP } \\
\text { suite }\end{array}$ \\
\hline R2 & $\otimes$ & $\otimes$ & $\begin{array}{l}\text { Networking technologies of the IP } \\
\text { suite and data download } \\
\text { frameworks e.g. .NET framework }\end{array}$ \\
\hline R3 & $\otimes$ & $\otimes$ & $\begin{array}{l}\text { Graphics standards and data } \\
\text { download frameworks e.g. Open } \\
\text { GL ES; .NET framework }\end{array}$ \\
\hline R4 & $\chi$ & $\chi$ & - \\
\hline R5 & $\otimes$ & $\otimes$ & $\begin{array}{l}\text { Networking technologies of the IP } \\
\text { suite and Graphics standards e.g. } \\
\text { Open GL ES }\end{array}$ \\
\hline R6 & $\otimes$ & $\otimes$ & $\begin{array}{l}\text { Graphics utilities and networking } \\
\text { technologies of the IP suite }\end{array}$ \\
\hline R7 & $\chi$ & $\otimes$ & $\begin{array}{l}\text { Handhelds and networking } \\
\text { technologies of the IP suite }\end{array}$ \\
\hline R8 & $\chi$ & $\otimes$ & $\begin{array}{l}\text { Networking technologies of the IP } \\
\text { suite }\end{array}$ \\
\hline R9 & $\chi$ & $\otimes$ & $\begin{array}{l}\text { Adaptation platforms, e.g. the } \\
\text { Windows Sensor and Location } \\
\text { platform }\end{array}$ \\
\hline R10 & $\chi$ & $\chi$ & - \\
\hline R11 & $\chi$ & $\otimes$ & $\begin{array}{l}\text { Networking technologies of the IP } \\
\text { suite }\end{array}$ \\
\hline R12 & $\chi$ & $\otimes$ & $\begin{array}{l}\text { nVidia mobile processor/ storing } \\
\text { data in memory chunks }\end{array}$ \\
\hline R13 & $\chi$ & $\chi$ & - \\
\hline R14 & $\chi$ & $\chi$ & - \\
\hline R15 & $\chi$ & $\otimes$ & $\begin{array}{l}\text { Transcoding/ Windows sensor } \\
\text { and location platform }\end{array}$ \\
\hline
\end{tabular}

characteristic requirements $R 1, R 2, R 3, R 5$ and $R 6$ will eventually be fulfilled if the proposed conceptual framework is partially or fully implemented - by associating these requirements to some elements of the proposed framework as well as to some of the existing communications and networking solutions, as shown in Table 2. It is difficult though, at this stage, to verify or speculate if characteristic requirements $R 4$, $R 7, R 8, R 9, R 10, R 11, R 12, R 13, R 14$ and $R 15$ have been fulfilled or will be fulfilled later on. For these characteristic requirements to be verified, low-level details of the environment need to be known. Overall, the current implementation of the environment (i.e. in the form of framework) only gives a basic conceptual structure and sketchily shows how the visualization environment will be like, but cannot be used to fully verify fulfillment of these characteristic requirements.

It is important to note, however, that the review carried out to some extent has helped us to reflect critically on the suitability of the proposed conceptual framework and on the completeness of the requirements. Obviously it is important to incrementally 


\section{Adapt image to dsplay convention (K1) Interfaceprogramfor server $(K 3)$ Capturedynamic effects(K6) \\ Datasharing $(K 5)$ \\ Pertaintofunctionality need $(K 2)$

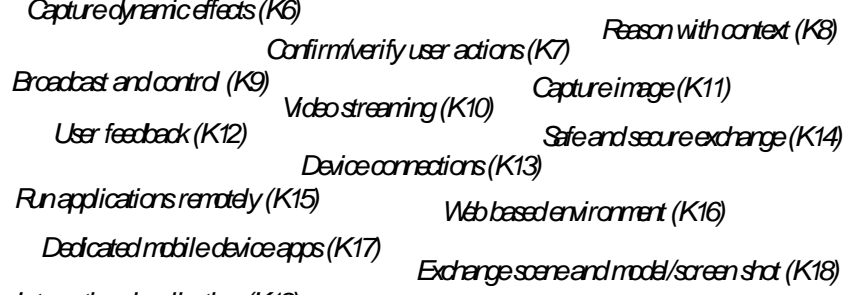

\section{Interactive visulization (K19) Content management (K21) \\ Devices adapting to comecting applications (K20) \\ Interfaceprogramfor visualization device(K22)}

Capture verbal comminications \& context (K23)

STrart adaptations (K25)

Capturegestures(K26)

Ramatemodding(K24)

Fig.7. Phrases or keywords used by focused group participants to describe the desirable features of the heterogeneous remote product visualization environment.

verify requirements and to validate spontaneous hypotheses in the subsequent stages of the development process as well, e.g. at the: architecture development stage (to, e.g., inspect the adequacy of functionality allocations); at the design stage (to evaluate algorithms and features) and after implementation (e.g., to try out prototypes as well as to study the acceptability and usability of the implementations by involving users).

\subsection{Focus Group Research}

Focus group interviews [38] were held in several occasions to seek opinions, perceptions and attitudes of various experts and possible future users towards the idea of developing a heterogeneous remote product visualization environment and using it to support various product development activities. The special feature of focus-group interviews is that they are more dynamic and generate a wide range of rich data through interactions with interviewees. Therefore, the purposes of holding focus group interviews in addition to the review described in Section 6.1 were to: (i) explore and to establish whether or not the requirements have correctly and comprehensively been formulated, (ii) determine the completeness of the framework, and to (iii) explore potential implementation solutions and identify possible application areas.

Nine subjects participated in the interviews. Five participants were design researchers with IT and computer aided design engineering background, and four were industrial design engineering graduate students. Their background ensured that they would have something to say about the discussion topics. Separate sessions were held informally and were built up as follows. In the beginning of each informal session, participants were informed about the idea of developing a heterogeneous remote product visualization environment that includes mobile communication devices, standard office based computers, and high-end visualization systems. The discussions were streamlined and guided under the following discussion statements: (1) using office-based visualization technologies

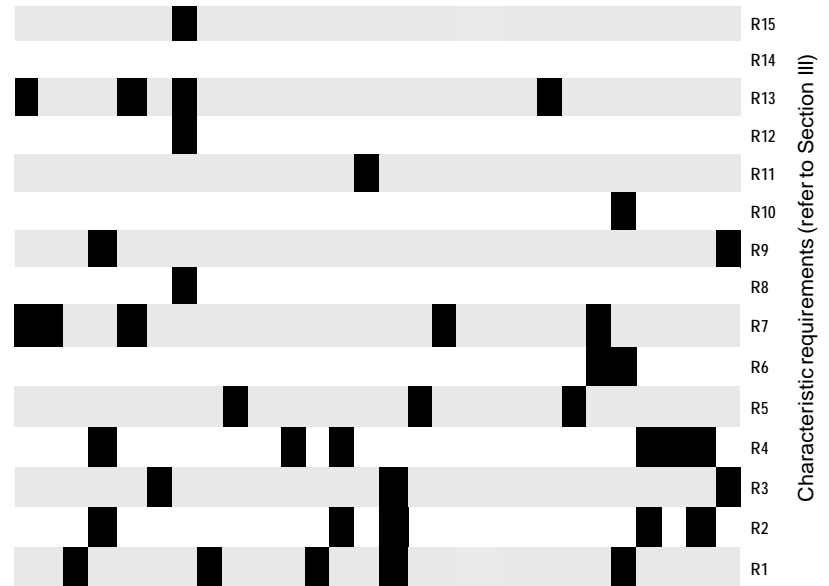

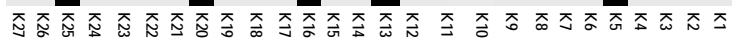

Phases/keywords (refer to Fig. 7)

Fig. 8. Aggregation of the associations of keywords and characteristic requirements.

and handheld portable devices jointly would be a proper way of designing, visualizing and exchanging knowledge among members of product development teams; (2) portable and non-portable communications and computing devices and systems would provide proper means for undertaking tasks efficiently in product development processes and for shortening times needed to finalize tasks in distributed product development processes; (3)a heterogeneous remote product visualization environment must fulfill certain basic characteristic requirements; (4) some existing solutions in the forms of hardware technologies, codes and networking solutions can be adopted and applied. Discussions were carried out in an interactive way and participants were free to discuss various issues related to the above listed topics. The sessions' durations ranged from about half an hour to one hour. The first 5-10 minutes of each session were spent on explaining how the envisioned environment might be like and the remaining time for open discussions. In each session, detailed notes on what was said were taken to allow analysis to be carried later on. This ensured that important points were not missed. These focus group discussions continued until a clear pattern emerged and discussions seemed to produce no significant new information.

The information collected from these focus group interviews was analyzed. The analysis essentially involved identifying and listing down the words and phrases used by focused group interviewees to describe the desirable features of the heterogeneous remote product visualization environment. Fig. 7 shows the keywords derived from the discussions. These keywords were then closely cross-examined to determine if they relate to the requirements for the envisioned heterogeneous remote product visualization environment presented and discussed in Section III. The associations of keywords and requirements were aggregated as shown in Fig. 8. It was found that the previously formulated requirements to a large extent sufficiently describe most of the keywords used by focused group interviewees when describing the desirable features of the heterogeneous remote product visualization 
environment. $89 \%$ of the keywords could be associated directly with the requirements. Only three keywords (i.e., K9 broadcast and control, K10 - video streaming, and K18 exchange scene and model/screen shot) - all of them related to some particular low level implementation details - could not be associated with the requirements. These findings verify the correctness of the requirements and, in a way, validate the framework created based on these requirements.

\section{SUMMARY AND CONCLUSIONS}

This paper presents the results from an on-going research that focuses on the challenge of providing suitable visual information to members of product development teams equipped with dissimilar visualization devices. Characteristic requirements for a heterogeneous remote product visualization environment have been formulated and a conceptual framework of the environment has been developed. Preliminary evaluation has also been carried out to determine the validity of the requirements and the completeness of the proposed framework. It has been shown that the proposed conceptual framework addresses the demands and challenges of remote product visualization. It provides a technological roadmap for implementation of an environment that supports remote product data and virtual models sharing, facilitates real-time image-based collaboration among members of product development teams, and fosters stakeholder's remote participation in solutions development in various activities regardless of their geographical locations.

However, there are still many difficult challenges that must be addressed in order to achieve a fully operational prototype of the prospective heterogeneous remote product visualization environment. Most of these challenges relate to enhancement of handheld devices. These include, for instance, the challenges posed by: (a) limited computing capabilities and local storage capacities of handheld devices; (b) relatively small screen sizes - which makes it difficult, for instance, to compare documents or to view contents of large folders, large images or long lists of search results; (c) limited interactivity - which makes it hard to edit graphical representations or to create product models; (d) diverse display and networking technology solutions - which makes it difficult, for instance, to carry over display contents from one device to another dissimilar device;(e) limited bandwidth [40], and (f) limited battery power. Power management in portable handheld devices is particularly critical for increasing the operational time of these devices, which typically have limited space for accommodating resources like batteries. It is, however, important to note that the existing handheld devices belong to a generation of technologies that have not specifically been developed for product visualization. In general terms, realization of a fully operational heterogeneous remote product visualization environment and ensuring smooth performance is a very complex challenge. Extensive research still needs to be carried out to overcome many challenges, including those mentioned above.

\section{REFERENCES}

[1] J. Fukai, K, Amafuji, H, Murata, Y. Color and high resolution head-mounted display. Proc. of SPIE 1994, 6 - 10 February 1994, San Jose, CA, USA, pp. 317-324.

[2] W. Buxton, G. W. Fitzmaurice, HMDs, Caves, and Chameleon: A Human-Centric Analysis of Interaction in Virtual Space, Computer Graphics, 32(4), 1998, p. 64-68.

[3] N. A. Dodgson, Autostereo displays: 3D without glasses, EID 97 (Electronic Information Displays), Esher, Surrey, 18-20 Nov 1997.

[4] M. Halle, Auto stereoscopic displays and computer graphics Computer Graphics, ACM SIGGRAPH, 31(2), p. 58-62, May 1997.

[5] C. Tsao, Method and apparatus for displaying volumetric 3D images, United States Patent 6765566, 2004.

[6] P. Hariharan, Optical Holography: principles, techniques, and applications, Cambridge Univ. Press, 1984.

[7] M. Lucente, Interactive Three-Dimensional Holographic Displays: Seeing the Future in Depth, ACM SIGGRAPH Computer Graphics, 31 (2), May 1997, pp. 63-67.

[8] E. Z. Opiyo, I. Horváth, Towards an interactive spatial product visualization: a comparative analysis of prevailing 3D visualization paradigms, Int. Journal of Product Development, 11(1/2), pp. 4-24, 2010.

[9] S. Zhang, W. Shen, and H. A. Ghenniwa, review of Internet-based product information sharing and visualization, Computers in Industry, 54 (1), p.1-15, May 2004.

[10] M.R. Cutkosky, R.S. Engelmore, R.E. Fikes, M.R. Genesereth, T.R. Gruber, W.S. Mark, J.M. Tenenbaum, J.C. Weber PACT: an experiment in integrating concurrent engineering systems, IEEE Computer 26 (1) pp. 28-37, 1993.

[11] G. Toye, M. Cutkosky, L. Leifer, J. Tenenbaum, J. Glicksman, SHARE: a methodology and environment for collaborative product development, in: Proc. of 2nd Workshop on Enabling Technologies: Infrastructure for Collaborative Enterprises, IEEE Computer Society Press, 20-22 April 1993, Morgantown, WV , USA, pp. 33-47.

[12] U. Roy, S.S. Kodkani, Product modeling within the framework of the World Wide Web, IIE Transactions, 31 (7), pp. 667-677, 1999.

[13] S. Rajagopalan, J.M. Pinilla, P. Losleben, Q. Tian, S.K. Gupta Integrated design and rapid manufacturing over the Internet, in: Proc. of the 1998 ASME DETC/CIE. Atlanta, GA, September 13-16, 1998, Paper No. DETC98/CIE-5519),

[14] G.D.F. Pahng, S. Bae, D. A. Wallace Web-based collaborative design modeling environment, in: Proc. of the IEEE Workshops on Enabling Technologies Infrastructure for Collaborative Enterprises (WET ICE98), pp. 161-167, 1998.

[15] K. Cheng, P.Y. Pan, D.K. Harrison, Web-based design and manufacturing support systems: implementation perspectives, Int. Journal of Computer Integrated Manufacturing 141 (1) pp. 14-27, 2001.

[16] A. Xiao, H.J. Choi, R. Kulkarni, J.K.. Allen, D. Rosen, F. Mistree, S.C. A. Feng, Web-based distributed product realization environment, in: Proc. of the 2001 ASME DETC/CIE Conf., Pittsburgh, PA, USA, September 9-12, 2001, paper No. DETC2001/CIE-21766.

[17] G. Q. Huang, K.L. Mak, WeBid: a Web-based framework to support early supplier involvement in new product development, Robotics and Computer Integrated Manufacturing 16 (2/3) pp. 169-179, 2000.

[18] J. Y. Kim, S.B. Han, Web-enabled feature-based modeling in a distributed design environment, in: Proc. of the 1999 ASME DETC/CIE. Conf., Las Vegas, NV, USA, September 12-15, 1999 Paper No. DETC99/DFM-8941.

[19] Raje, R. R., Boyles, M., and Fang, F. CEV: Collaborative Environment for Visualization Using Java RMI, Concurrency: Pract. Exper., Vol. 10(11-13), 1079-1085, 1998.

[20] P.A. Rodgers, A.P. Huxor, Caldwell N.H.M. Design support using distributed Web-based AI tools, Research in Engineering Design 11 (1), 31-44, 1999.

[21] Z. Zdrahal, J. Domingue, The World Wide Design Lab: an environment for distributed collaborative design, in: Proc. of 1997 Int. Conf. on Engineering Design, Tampere, 1997.

[22] R.H. Allen, S. Nidamarthi, S.P. Regalla, R.D. Sriram, Enhancing collaboration using an Internet integrated workbench, in: Proc. of the 1999 ASME Design Engineering Technical Conf., Las Vegas, NV, USA, September 12-15, 1999 Paper No. DETC99/DAC-8573.

[23] M. Klein, Capturing geometry rationale for collaborative design, in: Proc. of the IEEE Workshops on Enabling Technologies Infrastructure for Collaborative Enterprises (WET ICE97), 1997, pp. 24-28. 
[24] B. Gerritsen, I. Horváth, The upcoming and proliferation of ubiquitous technologies in products and processes, Proceedings of the TMCE 2010, April 12 - 16, 2010, Ancona, Italy, pp. 551 - 564.

[25] F.S. Tsai, W. Han, J. Xu, H.C. Chua, Design and development of a mobile peer-to-peer social networking application, Expert Systems with Applications, 36 (8), pp.11077-11087, 2009.

[26] R. Schollmeier, A Definition of Peer-to-Peer Networking for the Classification of Peer-to-Peer Architectures and Applications, Proc. of the First Int. Conf. on Peer-to-Peer Computing, IEEE, Linkoping , Sweden, pp. 101-102, 27-29 Aug. 2001.

[27] T. Mørkved, Peer-to-Peer Programming with Wireless Devices, M.Sc. Thesis, University of New South Wales \&Agder University College, 2005.

[28] S. Counts, terHofte, H., \& Smith, I. Mobile social software: Realizing potential, managing risks. In Proc. of the workshop at the Conf.on human factors in computing systems (CHI 06), Montréal, Québec, Canada. pp. 1703-1706, April 22-27, 2006.

[29] I. Smith, Social-mobile applications, Computer 38 (4), pp. 84-85, 2005.

[30] E. Harjula, , Ylianttila, M., Ala-Kurikka, J., Riekki, J., \&Sauvola, J. Plug-and-play application platform: Towards mobile peer-to-peer. In Proc. of the third Int. Conf. on mobile and ubiquitous multimedia, College Park, Maryland, USA, October 27 - 29, 2004, Vol. 83 pp. 63-69, 2004.

[31] A. Duizer, Sensor and Location platform Windows 7, Microsoft.NET magazine, pp. 10 - 13, September 2009.

[32] H. Sun, Xuemin Chen, X., and Chiang, T. Digital Video Transcoding for Transmission and Storage, New York, CRC Press, 2005.

[33] S. Eminsoy, Dogan, S., Kondoz, A. M. Transcoding-Based Error-Resilient Video Adaptation for 3G Wireless Networks, EURASIP Journal on Advances in Signal Processing, Vol. 2007 (2007), Article ID 39586, 13 pages, doi:10.1155/2007/39586.

[34] A. Butter and D. Pogue, Piloting Palm, John Wiley \& Sons, New York, 2002.

[35] E. Opiyo, Z.Facilitating the Development of Design Support Software by Abstract Prototyping, Ph.D. thesis. Delft University of Technology, Delft, the Netherlands, 2003.

[36] E. Z. Opiyo, Horváth, Vergeest, J. S. M., "Quality Assurance of Design Support Software: Review of the state of the art", Computers in Industry Journal, 49(2), pp. 195-215, 2002.

[37] E. Z. Opiyo, Horváth, I. and Vergeest, J. S. M., "Extending the Scope of Quality Assurance of CAD Systems - Putting Underlying Engineering Principles, Theories and Methods on the Spotlight”, Journal of Computing and Information Science in Engineering (JCISE) Vol. 9, Issue 2, June 2009, 024502-1 - 024502-7 DOI:10.1115/1.3130778.

[38] F. Rabiee, Focus-group interviews and data analysis, Proc. of the Nutrition Society, 63, 655-660, 2004.

[39] J. Nielsen and R. L. Mack, Usability Inspection Methods, New York City, NY:John Wiley \& Sons, 1994.

[40] I. Horváth, Z. Rusák, E. Z. Opiyo, A. Kooijman, Towards Ubiquitous Design Support. In Proc. of the ASME Design Engineering Technical Conf., San Diego, CA, USA, August 30 - September 2, 2009, Paper No. DETC2009-87573.

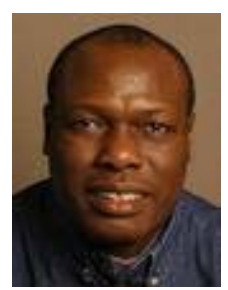

Eliab Z. Opiyo is an Assistant Professor at the Faculty of Industrial Design Engineering, Delft University of Technology, in the Netherlands. He holds a Ph.D. degree (Computer Aided Engineering) (2003) from Delft University of Technology, the Netherlands and a M.Sc. degree (Computer Aided Design and Manufacturing) (1992) from Cranfield University, United Kingdom. His current research interests include product visualization, applications of mobile and ubiquitous technologies in product development, prototyping, and development of design support systems.

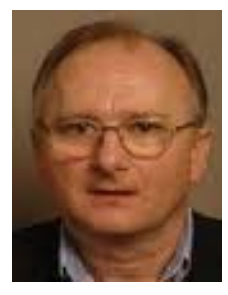

Imre Horváth is a Full Professor of Computer Aided Design and Engineering at the Faculty of Industrial Design Engineering, Delft University of Technology, in the Netherlands. He earned his Dr. univ. title (1987) and Ph.D. title (1994) from TU Budapest in Hungary. His current research interests include design application of mobile and ubiquitous technologies, systemization of design research, affordance based innovation, and teleconferencing-based active learning. 